\title{
THE POSITION OF $C$-SETS IN SEMIGROUPS ${ }^{1}$
}

\author{
A. D. WALLACE
}

A continuum is a compact connected Hausdorff space. If $X$ is a continuum, then $C \subset X$ is a $C$-set if (i) $C \neq X$, (ii) for each subcontinuum $A \subset X$ that meets $C$ we have either $A \subset C$ or $C \subset A$. Thus a composant of a solenoid is a $C$-set.

A $m o b$ is a Hausdorff space together with a continuous associative multiplication. A clan is a compact connected mob with (two-sided) unit, denoted by $u$. In what follows we assume that $S$ is a mob.

(*) Assume that $S$ is compact. Let $E$ be the set of idempotents of $S$ and let $K$ be the minimal ideal of $S$ [4]. We know that each $e \in E$ is contained in a maximal subgroup $H(e)$ of $S$ and that no two such maximal subgroups intersect $[8$, Theorem 1]. Moreover ([1] and [8, Theorem 3]),

$$
K=\bigcup\{H(e) \mid e \in E \cap K\},
$$

and, if $e \in E$, then $e \in K$ if and only if $H(e)=e S e$. Finally, $K=\bigcup\{L \mid L$ $\in \mathcal{L}\}$ where $\mathcal{L}$ is the set of minimal left ideals of $S$ and $L \in \mathcal{L}$ if and only if $L=S x$ for some $x \in K[1]$.

We refer to the above paragraph as $\left(^{*}\right)$. Its content is essential for the proofs of most of the theorems below. In some cases we may cite $\left({ }^{*}\right)$ and use instead the left-right dual of an assertion in $\left(^{*}\right)$.

Our purpose in this note is to examine the position of $C$-sets in clans relative to $K$ and to the sets $H(e), e \in E$. We show, for example, that if $C$ is a $C$-set meeting $H(e) \neq\{e\}$, then $C \subset H(e)$. As noted earlier, topological groups may contain $C$-sets and it is easy to give examples of clans which are not groups and which contain $C$-sets. The results here are more decisive than those in [6].

The following lemma may be presumed to lie in the public domain, and we are unable to give any references.

Lemma 1. Let $X$ be a continuum and let $C$ be a $C$-set of $X$. Then $C$ is connected and contains no inner points.

The proof of the lemma consists of repeated applications of the familiar result that, if $X$ is a continuum and if $U$ is an open subset with $U^{*} \backslash U \neq \square$, then each component $C$ of $U$ satisfies $C^{*} \cap\left(U^{*} \backslash U\right)$ $\neq \square$.

Received by the editors September 26, 1954.

1 This work was done under Contract N7-onr-434, Task Order III, Navy Department, Office of Naval Research. 
If $X$ is a space and if $\left\{A_{\lambda} \mid \lambda \in \Lambda\right\}$ is a family of subsets of $X$ indexed by a directed set $\Lambda$, then we define two subsets of $X$, sup $A_{\lambda}$ and $\inf A_{\lambda}$, as follows: $x \in \sup A_{\lambda}\left\{x \in \inf A_{\lambda}\right\}$ if, for each open set $U$ about $x$, there exists a cofinal $\{$ residual subset $\Lambda(U) \subset \Lambda$ such that $U \cap A_{\lambda} \neq \square$ for each $\lambda \in \Lambda(U)$. We write $\lim A_{\lambda}=A$, or $A_{\lambda} \rightarrow A$ if $\inf A_{\lambda}=A=\sup A_{\lambda}$. If $\Lambda$ is the set of positive integers, then this notion of convergence is the usual one, given for example in detail in Kuratowski [3]. We shall suppose that the reader can supply the simple unstated results that we need concerning this notion.

Lemma 2. Let $S$ be a compact mob, and let $\left\{A_{\lambda} \mid \lambda \in \Lambda\right\}$ and $\left\{B_{\lambda} \mid \lambda \in \Lambda\right\}$ be two families of subsets of $S$ based on the same directed set $\Lambda$. If $A_{\lambda} \rightarrow A$ and $B_{\lambda} \rightarrow B$, then $A_{\lambda} \cdot B_{\lambda} \rightarrow A \cdot B$ [5].

As in [2, Lemma 2], we may prove

Lemma 3. If $S$ is a continuum with left unit and if $L$ is a left ideal of $S$, then $K \cup L$ is connected.

THEOREM 1. Let $S$ be a continuum with left unit, let $L$ be a closed left ideal of $S$, and let $C$ be a $C$-set of $S$. If $C$ meets $K \cup L$ then $C \subset K \cup L$ (cf. [6, Theorem 1]).

Proof. By Lemma 3 we know that $K \cup L$ is a continuum. We may assume that $K \cup L$ is a proper subset of $C$. Let $p \in C \backslash(K \cup L)$ and let $U$ be an open set about $p$ such that $U^{*}$ does not meet $K \cup L$. Let $M$ be the union of all left ideals of $S$ contained in $S \backslash U^{*}$. Then $M$ is an open [2, Lemma 1 ] left ideal which is connected by Lemma 3. Now $M^{*}$ is a continuum not containing $p$ and intersecting $C$. Hence $M^{*} \subset C$, contrary to the fact that, by Lemma $1, C$ contains no inner points.

THEOREM 2. If $S$ is a clan and if $K$ is a $C$-set, then $K$ is a maximal subgroup of $S$.

Proof. If $S$ is a group then $K=S$ and the result follows. If $S$ is not a group then $S \backslash H(u)$ is an ideal [2, Theorem 4] so that $u$ is not in $K$ and $S \backslash K$ is not void. Recall that $S$ is connected and that $K$ has no inner points by Lemma 1 . Let $C$ be an idempotent in $K\left(\right.$ see $\left({ }^{*}\right)$ ) and let $\left\{a_{\lambda} \mid \lambda \in \Lambda\right\}$ be a directed set of points of $S \backslash K$ with $a_{\lambda} \rightarrow e$. Now $a_{\lambda} \in a_{\lambda} S$ and $a_{\lambda} S$ meets $K$ because any right ideal meets any ideal. Since $K$ is a $C$-set and $a_{\lambda} \in S \backslash K$ we must have $K \subset a_{\lambda} S$ for each $\lambda$. Thus $K \subset \cap\left\{a_{\lambda} S \mid \lambda \in \Lambda\right\} \subset \lim a_{\lambda} S=e S$, by Lemma 2. Thus $K \subset e S$ and dually $K \subset S e$ so that $K \subset e S e=e S \cap S e$. Now $e \in K$ gives $e S e \subset K$ and $e S e=H(e)\left(\right.$ see $\left.\left({ }^{*}\right)\right)$ and thus $K=H(e)$.

This result may fail if $S$ has only a left unit. 
Theorem 3. If $S$ is a clan, if $e \in E$, and if $C$ is a $C$-set meeting $H(e) \neq\{e\}$, then $C \subset H(e)$.

Proof. Since $H(e) C e S e=e S \cap S e$ we know that $C$ intersects both $S e$ and $e S$.

(A) Let $C \cap K=\square$. Now eS is a continuum which intersects $K$ so that, of the two possibilities $C \subset e S$ and $e S \subset C$, we must have the former. Similarly $C \subset S e$ so that $C \subset e S e$. Now $e S e$ is a clan with unit $e$ and $H(e)$ is the maximal subgroup of $e S e$ containing $e$. By Theorem 2 of [6] and Lemma 1 it follows that $C \subset H(e)$.

(B) Let $C \cap K \neq \square$ so that $C \subset K$ by Theorem 1 . Thus $H(e)$ meets $K$ and so $H(e) \subset K$ since $H(e)$ is a subgroup of $S$. Since $H(e) \subset e S \cap S e$ both $e S$ and $S e$ intersect $C$. There are four possibilities which we state and consider.

(i) $e S \cup S e \subset C$. We know (see $\left({ }^{*}\right)$ ) that each $L \in \mathcal{L}$ meets $e S$. For any such $L \neq S e$ we have $L \subset C$ since $L$ is a continuum ( $L=S x$ for some $x \in K$, see $\left({ }^{*}\right)$ ) and $C \subset L$ would give $L \cap S e \neq \square$, contrary to the fact that $L$ and $S e$ (see $\left({ }^{*}\right)$ ) are minimal left ideals. Since $K$ is the union of all the minimal left ideals of $S$ (see $\left(^{*}\right)$ ) we know that $K \subset C$ and thus $C \subset K$ by Theorem 1 . By Theorem $2, K$ is a maximal subgroup of $S$, i.e., $K=H(e)$, so that $C \subset H(e)$.

(ii) $e S \subset C \subset S e$. With the notation of $\left(^{*}\right)$ each $L \in \mathcal{L}$ intersects $e S$ and thus $S e$ and hence $L=S e$. It follows by $\left(^{*}\right)$ that $K=S e$. By the dual of Theorem 1 of [7], and that part of the proof of Theorem 4 of [7] on the middle of page 53, we know that there is a topological isomorphism $\phi: K \rightarrow H(e) \times(E \cap K)$. If $E \cap K$ is degenerate then $K=H(e)$ and $C \subset H(e)$. If $E \cap K$ contains more than one element then $(H(e)$ $\neq\{e\}$ by assumption) the cartesian product of two nondegenerate continua contains a $C$-set, $\phi(C)$. Now a somewhat dull argument shows that $\phi(C)$ is a point and thus $C$ is a point and hence $C \subset H(e)$.

(iii) $S e \subset C \subset e S$. This is the dual of (ii).

(iv) $C \subset S e$ and $C \subset e S$. Then $C \subset e S \cap S e=e S e=H(e)$ since $e \in K$ and using $\left({ }^{*}\right)$.

Although our last theorem does not involve $C$-sets, it fits easily into the pattern of this note.

With the aid of Theorem 4 and Lemma 3 of [2] as well as the argument used in the proof of these results we can prove

LEMMA 4. If $S$ is a clan with unit $u$ and if $H(u)$ contains an inner point, then $H(u)=S$.

THEOREM 4. If $S$ is a continuum, if $e \in K$, and if $H(e)$ has an inner point, then $H(e)=K$. 
Proof. It is clear that $e S e$ is a clan with unit $e$, that $H(e)$ is the maximal subgroup of $e S e$ containing its unit and that $H(e)$ has inner points relative to $e S e$. Thus $H(e)=e S e$ by Lemma 4 and by $\left(^{*}\right)$ $H(e) \subset K$. Suppose that $H(e) \neq K$. By Lemma $3, K$ is connected so that there is a directed set of points $\left\{x_{\lambda} \mid \lambda \in \Lambda\right\}$ of $K \backslash H(e)$ with $x_{\lambda} \rightarrow x \in H(e)$. It follows from Lemma 2 that $x_{\lambda} S x_{\lambda} \rightarrow x S x=e S e=H(e)$. Since $H(e)$ contains an inner point we know by definition that $H(e) \cap x_{\lambda} S x_{\lambda} \neq \square$ for some $\lambda \in \Lambda$. Now $x_{\lambda} \in H(f)$ for some $f \in E \cap K$ (see $\left(^{*}\right)$ ) and $H(f)=f S f$ readily gives $H(f)=x_{\lambda} S x_{\lambda}$. Thus $H(e)$ meets $H(f)$ and, by $\left({ }^{*}\right), H(e)=H(f)$ contrary to the fact that $x_{\lambda} \in S \backslash H(e)$ and $x_{\lambda} \in H(f)$.

Remark. With the aid of Lemma 2 and $\left({ }^{*}\right)$ it easily follows that $\mathcal{L}$ is a continuous decomposition of $K$ in this sense: If $\left\{x_{\lambda} \mid \lambda \in \Lambda\right\}$ is in $K$, if $x_{\lambda} \rightarrow x$, and if $L_{\lambda}$ is the member of $\mathcal{L}$ containing $x_{\lambda}$ then $L_{\lambda} \rightarrow L$, the member of $\mathcal{L}$ containing $x$. Thus if $\phi: K \rightarrow \mathcal{L}$ is the natural function and if we give $\mathcal{L}$ the usual topology then $\phi$ is a continuous open function and $\mathcal{L}$ is a compact Hausdorff space. Of course we are supposing that $S$ is compact. A similar result is obtained using the sets $H(e), e \in E \cap K$.

\section{REFERENCES}

1. A. H. Clifford, Semigroups containing minimal ideals, Amer. J. Math. vol. 70 (1945) pp. 521-526.

2. R. J. Koch and A. D. Wallace, Maximal ideals in compact semigroups, Duke Math. J. vol. 21 (1954) pp. 681-686.

3. C. Kuratowski, Topologie I and II, Warsaw, 1948 and 1950.

4. K. Numakura, On bicompact semigroups, Math. J. Okayama University vol. 1 (1952) pp. 99-108.

5. W. L. Strother, Continuity for multi-valued functions, Tulane University dissertation, 1952.

6. A. D. Wallace, Indecomposable semigroups, Math. J. Okayama University vol. 3 (1953) pp. 1-3.

7. - Cohomology, dimension and mobs, Summa Brasiliensis Mathematicae vol. 3 (1953) pp. 43-54.

8. (1953) pp. 335-336.

The Tulane University of Louisiana 